\title{
Variations in Zebra Chip Disease Expression and Tuber Biochemistry in Response to Vector Density
}

\author{
Arash Rashed, Christopher M. Wallis, Fekede Workneh, Li Paetzold, and Charles M. Rush
}

First author: University of Idaho, Department of Plant, Soil, and Entomological Sciences, Aberdeen Research \& Extension Center, Aberdeen 83210; second author: United States Department of Agriculture-Agricultural Research Service, San Joaquin Valley Agricultural Sciences Center, Crop Diseases, Pests and Genetics Research Unit, 9611 S. Riverbend Ave, Parlier, CA 93648; and third, fourth, and fifth authors: Texas A\&M Agrilife Research and Extension, 6500 Amarillo Blvd W., Amarillo 79106. Accepted for publication 10 April 2016.

\begin{abstract}
Rashed, A., Wallis, C. M., Workneh, F., Paetzold, L., and Rush, C. M. 2016. Variations in zebra chip disease expression and tuber biochemistry in response to vector density. Phytopathology 106:854-860.

Zebra chip (ZC) disease, caused by 'Candidatus Liberibacter solanacearum', which is transmitted by the potato psyllid, has negatively affected potato production in the United States for over a decade. The present study was conducted to evaluate the affect of the number of bacteriliferous psyllids on ' $C a$. L. solanacearum' titer, levels of amino acids, carbohydrates, phenolics, and, subsequently, symptom severity in potato tubers. 'Red La Soda' and 'Russet Norkotah' potato were planted in the field and later inoculated with ' $\mathrm{Ca}$. L. solanacearum' using 5,10 , and 30 bacteriliferous potato psyllids. In both cultivars, the increase in the number of psyllids resulted in elevated ' $C a$. L. solanacearum' titer and symptom severity. In the cases of amino acids

and reducing sugars, responses to vector density appeared to be cultivar specific. Overall, phenolic compounds showed a consistent increase following infection, a response that, in most cases, was positively correlated with the number of infective psyllids. Results quantified the impact of the number of infective psyllids on ' $\mathrm{Ca}$. L. solanacearum' titer and biochemical content of infected tubers previously shown to be correlated with the degree of symptom expression. Managing vector numbers is currently the only effective approach for minimizing losses to $\mathrm{ZC}$ in grower's fields. However, our findings on physiological responses to vector density suggest that, in combination with chemical control, development of more tolerant or resistant potato genotypes, with emphasis on interrupting pathways that are associated with increased phenolic activity levels, may lead to more sustainable management of $\mathrm{ZC}$ in the future.
\end{abstract}

Zebra chip (ZC) is an affliction of potato that has been affecting U.S. potato production for more than a decade. $\mathrm{ZC}$ is associated with a vector-borne phloem-limited fastidious bacterium, 'Candidatus Liberibacter solanacearum' (Wen et al. 2009). The potato psyllid Bactericera cockerelli (Hemiptera: Triozidae) is known to transmit ' $C a$. L. solanacearum' not only in potato but also in a wide range of other solanaceous host plants (Hansen et al. 2008; Munyaneza 2012; Munyaneza et al. 2007). In potato, foliar symptoms of ZC resemble those of other conditions such as 'purple top' and 'psyllid yellows' and include leaf curling, yellowing, leaf-scorch, shortened internodes, and the formation of aerial tubers, which often result in plant death (Munyaneza et al. 2007; Secor et al. 2009). However, the disease is primarily characterized by symptoms visualized in tubers that include browning of both freshly cut and fried tuber slices. Significant yield losses occur when infections occur prior to and during the tuber initiation stage (Rashed et al. 2014). However, late-season ' $\mathrm{Ca}$. L. solanacearum' infections just days before harvest also can lead to quality loss in infected tubers (Rashed et al. 2014; Rush et al. 2015). Both foliar and tuber symptoms are associated with changes in plant physiology, including shifts in phenolic, reducing sugar, and amino acid levels, and these have been reported to affect fresh and processed potato quality (Navarre et al. 2009; Rashed et al. 2013; Wallis et al. 2012, 2014, 2015a,b). Changes in phenolic compounds are associated with disease severity in freshly cut tuber slices, and the increase in reducing sugars and amino acids contributes to excessive browning of fried potato slices (Rashed et al. 2013; Wallis et al. 2012). The emerged burn patterns in

Corresponding author: A. Rashed; E-mail address: arashed@uidaho.edu potato following exposure to high temperatures has been associated with brown-colored acrylamide, resulting from the Maillarddriven reaction between free amino acids and reducing sugars (Stadler et al. 2002).

Potato psyllids are highly efficient in transmitting ' $\mathrm{Ca}$. L. solanacearum'. An individual psyllid vector of ' $\mathrm{Ca}$. L. solanacearum' would have between 30 and $70 \%$ chance of transmitting the pathogen to potato plants within $48 \mathrm{~h}$ (Rashed et al. 2012). As the number of feeding vectors increases, there would also be an increase in the amount of ' $C a$. L. solanacearum' inoculated into the plant tissue, an effect that has been shown to influence foliar symptom expression of the infected host by reducing the incubation time of the disease (Rashed et al. 2012). It is also conceivable that variations in the number of infective psyllids may also differentially affect plant biochemical components known to be correlated with the degree of symptom expression. How different densities of bacteriliferous psyllids affect quantity of plant biochemical components in infected plants and subsequent disease development is highly significant, especially with regard to late-season infections.

The present study was initiated to evaluate whether ' $C a$. L. solanacearum' titer and various biochemical compounds, including amino acids, phenolics, and carbohydrates, some of which have been associated with ZC symptom expression, vary in response to the number of bacteriliferous potato psyllid vectors. Because late-season infections are the norm in many of the major potato-producing regions of the United States, information on factors that could affect disease incubation period is of immediate value to potato farmers in those regions for making informed management decisions (Rush et al. 2015).

\section{MATERIALS AND METHODS}

Field experiment. Experiments were conducted at the Texas A\&M Agrilife Research and Extension Center in Bushland in two 
consecutive seasons, 2012 and 2013. Seed potato of 'Red La Soda' and 'Russet Norkotah' were planted in April 2011. All plants were covered with field cages of 100 by 100 by $80 \mathrm{~cm}$ (width by length by height) prior to emergence. To set up the field cages, Sunguard II fiberglass rods (Geotek Inc.) and netting 750 mesh (U.S. Global Resources) were used. Following emergence, plants were thinned to four plants, one close to every corner of the cage, approximately $50 \mathrm{~cm}$ apart.

Inoculations were conducted by releasing 5,10 , or 30 bacteriliferous potato psyllids from a ' $\mathrm{Ca}$. L. solanacearum'-positive colony maintained in the greenhouse at the base of a single plant within a cage. The greenhouse colony was reared on 'FL1867' potato (three to four plants) in bugdorm cages of 60 by 60 by $60 \mathrm{~cm}$ (BioQuip Products) for several generations. These potato psyllids were of the central haplotype (Swisher et al. 2012), originally collected from commercial fields in Texas. The ' $\mathrm{Ca}$. L. solanacearum' biotype of the greenhouse colony was determined to be a mixture of ' $\mathrm{Ca}$. L. solanacearum' A and 'Ca. L. solanacearum' B (Wen et al. 2012). Prior to plant infestation each year, a random sample of 10 potato psyllids was removed from the colony and checked for ' $\mathrm{Ca}$. L. solanacearum' using conventional polymerase chain reaction (PCR) and all individuals were found to be positive for ' $\mathrm{Ca}$. L. solanacearum'.

Inoculations were conducted 5 weeks after plant emergence. The plant at the point of release in each cage was designated as "plant one" and used in subsequent analyses for the sake of consistency. This was done because analyses of parallel studies with similar design indicated that psyllids have limited tendency to move once they alight on a host plant (Rashed et al. 2014). In 2012, there were five cage replicates per psyllid density treatment, per potato variety, as well as five noninfested control cages. In 2013, there were three cage replicates for every vector density per variety. Insects were allowed to feed for 1 week (inoculation access period), and then were sprayed with an insecticidal mix containing Movento (Bayer Crop Science), AgriMek, and Fulfill (Syngenta Crop Protection). Infections were allowed to develop for 8 weeks. At harvest, plants were evaluated for symptom severity based on a 0-to-3 scale (Rashed et al. 2013). Three to four randomly selected symptomatic tubers from plant one were sampled for ' $\mathrm{Ca}$. L. solanacearum' quantification. Slices of the same tubers were shipped to the United States Department of Agriculture-Agricultural Research Service (USDA-ARS) in Parlier, CA, for biochemical analyses.

' $C a$. L. solanacearum' extractions and quantifications were performed at the Texas A\&M AgriLife Research and Extension in Bushland. A slightly modified DNeasy Plant Mini Kit (Qiagen) protocol was used. Immediately following removal from $-80^{\circ} \mathrm{C}$ storage, tuber samples (approximately $100 \mathrm{mg}$ ) were ground in a homogenizer until they reached a homogeneous soup consistency. After the first set of buffers was added, the samples were vortexed for $30 \mathrm{~s}$ each and incubated at $65^{\circ} \mathrm{C}$ for $15 \mathrm{~min}$ to maximize yield.

Using an Applied Biosystems 7500 real-time PCR, a comparative cycle threshold method was used to quantify ' $\mathrm{Ca}$. L. solanacearum' in relation to a calibrator and an endogenous $18 \mathrm{~S}$ RNA control. The reaction mix consisted of TaqMan Universal Master Mix (Applied Biosystems), 0.3 $\mu \mathrm{M}$ forward primer $\mathrm{LsoF}$ ( $\mathrm{Li}$ et al. 2009), 0.3 $\mu \mathrm{M}$ reverse primer $\mathrm{HLBr}$ (Li et al. 2006), and $0.25 \mu \mathrm{M}$ HLBp TaqMan probe ( $\mathrm{Li}$ et al. 2006). The quantity of ' $\mathrm{Ca}$. L. solanacearum' was normalized to the endogenous reference, eukaryotic $18 \mathrm{~S}$ ribosomal RNA (VIC/MGB probe, primer limited; Applied Biosystems). Quantifications were in relation to a calibrator containing 50,000 genome copies. Multiple plates were analyzed at the same time, using the plate study function of ABI default analysis software.

Biochemical analyses were performed at the USDA-ARS San Joaquin Valley Agricultural Sciences Center in Parlier, CA. Each potato tissue sample was divided into two aliquots of at least $50 \mathrm{mg}$. One aliquot was extracted over $48 \mathrm{~h}$ at $4^{\circ} \mathrm{C}$ in methanol $(1: 10$ [wt/vol]; for example, $50 \mathrm{mg}$ of tuber tissue extracted in $500 \mathrm{ml}$ of methanol) for phenolic compound analysis using a Shimadzu highperformance liquid chromatograph (HPLC) (Wallis et al. 2012). A
Shimadzu HPLC equipped with a Supelco C-611 carbohydrate column (300 by $7.8 \mathrm{~mm}$ ) and a refractive index detector (RID-10) were used to analyze carbohydrates in the other aliquot, extracted over $48 \mathrm{~h}$ at $4^{\circ} \mathrm{C}$ in phosphate-buffered saline (PBS) buffer $(1: 10$ [wt/vol]) (Rashed et al. 2013). PBS buffer extracts of tuber samples had amino acids analyzed by a commercially available kit from Phenomenex, using a Shimadzu GC2010-FID gas chromatograph to identify and quantify volatile derivatives (Rashed et al. 2013).

Statistical analyses were performed in IBM SPSS (ver. 22). Density treatment, plant replicate, cultivar, and density-cultivar interaction were factors included in all initial generalized linear models. Plant replicate and the interaction terms were removed from the final model in a stepwise approach if the effect was nonsignificant $(P>0.05)$. Poisson distribution with a logarithmic link was used for symptom severity analyses. Noninfected controls were included in ' $\mathrm{Ca}$. L. solanacearum' and symptom severity analyses to avoid bias in conclusions. The noninfected controls were included in all biochemical comparisons. Multivariate analysis of variance (MANOVA) was used to analyze amino acids, phenolics, and reducing sugars in infected and noninfected controls. Psyllid density treatment and plant replicate were included as factors. In all analyses, plant replicate was removed if its effect was nonsignificant $(P>0.05)$. Results were presented separately for year and cultivar factors, where a significant cultivar-year interaction was detected. To control for multiple comparisons of the potentially dependent variables, MANOVA conclusions were drawn based on the Bonferroni adjusted level of significance $(\alpha)$. Because the correction also allows the possibility of false nonsignificance, all standard errors of actual means are presented in tables for clarity. Pearson correlation (Pearson corr.) was used to evaluate relationships between ' $\mathrm{Ca}$. L. solanacearum' titer and different biochemical components. Spearman rank $\rho$ correlation was used to quantify relationships between Symptom severity and ' $\mathrm{Ca}$. L. solanacearum' as well as the evaluated biochemical properties.

\section{RESULTS}

'Ca. L. solanacearum' titer and symptom severity in Red La Soda and Russet Norkotah. Analysis of variance revealed a significant effect of vector density on ' $\mathrm{Ca}$. L. solanacearum' quantity in tubers $\left(F_{2,171}=21.81, P<0.001\right)$. Overall, ' $C a$. L. solanacearum' titer in tubers infested with 5 potato psyllids was significantly lower than in plants infested with 15 and 30 psyllids (Fig. 1). The presence of a significant year-cultivar interaction term indicated that the differences in ' $C a$. L. solanacearum' quantities between the two varieties was not consistent across the 2 years $\left(F_{1,171}=30.35, P<\right.$ 0.001) (Fig. 1) even though, overall, Red La Soda had significantly higher titer levels $\left(F_{1,165}=4.76, P=0.030\right)$. Plant replicate $(P=$ $0.113)$ and psyllid density-cultivar $(P=0.510)$ interaction terms were excluded from the final model due to their nonsignificant effects.

Symptom severity was significantly affected by potato cultivar (Wald $X^{2}{ }_{1}=13.97, P<0.001$ ) because Red La Soda consistently expressed lower degrees of symptom severity than Russet Norkotah in both years. Plants infested with 5 potato psyllids expressed lower degrees of symptom severity than plants infested with 15 or 30 potato psyllids (Wald $X^{2}{ }_{2}=16.14, P=0.001$ ). Plant replicate (Wald $\left.X^{2}{ }_{4}=4.78, P=0.31\right)$ and psyllid density-cultivar $(P=0.956)$ were excluded from the final model. In both cultivars, symptom severity was positively correlated with titer levels (Red La Soda: Spearman $\rho=0.684, n=90, P<0.001$ and Russet Norkotah: $\rho=0.508, n=90$, $P<0.001)$.

Amino acids. Amino acid concentrations showed great variability because they were affected by all MANOVA parameters. Differences in amino acid levels between the two varieties in response to psyllid density and across years were detected (psyllid density-cultivar: Pillai's Trace $=0.505, F_{42,636}=3.06, P<0.001$; year-cultivar: Pillai's Trace $\left.=0.503, F_{14,210}=15.18, P<0.001\right)$; therefore, the two varieties were analyzed separately. 
In Red La Soda, there was a significant effect of psyllid density (Pillai's Trace $=0.955, F_{42,297}=3.30, P<0.001$ ), year (Pillai's Trace $=0.92, F_{14,97}=78.58, P<0.001$ ), and plant replicate (Pillai's Trace $\left.=1.06, F_{56,400}=2.56, P<0.001\right)$. Of the 14 amino acids tested, serine $\left(F_{3,110}=1.96, P=0.123\right)$, aspartic acid $\left(F_{3,110}=1.26\right.$, $P=0.291)$, asparagine $\left(F_{3,110}=2.04, P=0.112\right)$, and glutamine $\left(F_{3,110}=1.26, P=0.291\right)$ were compounds for which levels were not affected by psyllid density treatment. The noninfested controls generally had lower concentration of amino acids than those infested with bacteriliferous psyllids, especially the 15-psyllid and 30-psyllid treatments (Table 1).

Similar to Red La Soda, amino acid concentrations in Russet Norkotah were greatly affected by psyllid density (Pillai's Trace $=$ $\left.1.11, F_{42,294}=4.13, P<0.001\right)$, year (Pillai's Trace $=0.92, F_{14,96}=$ 75.50, $P<0.001$ ), and plant replicate (Pillai's Trace $=1.20$,
$\left.F_{56,396}=3.04, P<0.001\right)$. Aspartic acid $\left(F_{3,109}=0.556, P=0.645\right)$ and glutamic acid $\left(F_{3,109}=1.72, P=0.165\right)$ were the only amino acids for which levels were not affected by the psyllid density treatment in Russet Norkotah. Noninfested controls had significantly lower concentrations of amino acids compared with tubers infested with 15 and 30 bacteriliferous psyllids (Table 1).

In Red La Soda, asparagine $(\rho=-0.187, n=122, P=0.119)$, glutamic acid $(\rho=-0.005, n=122, P=0.96)$, and glutamine $(\rho=-0.063, n=122$, $P=0.559)$ were the only amino acids not affected by the degree of symptom severity. Levels of all remaining amino acids were positively correlated with symptom severity (all $P$ values $<0.005$ ). Unlike other amino acids, asparagine, aspartic acid, methionine, glutamic acid, and glutamine were the only amino acids for which levels were not affected by ' $\mathrm{Ca}$. L. solanacearum' titer (Pearson corr., all $P$ values $>0.171$ ) (Table 2). Levels of all remaining amino acids were
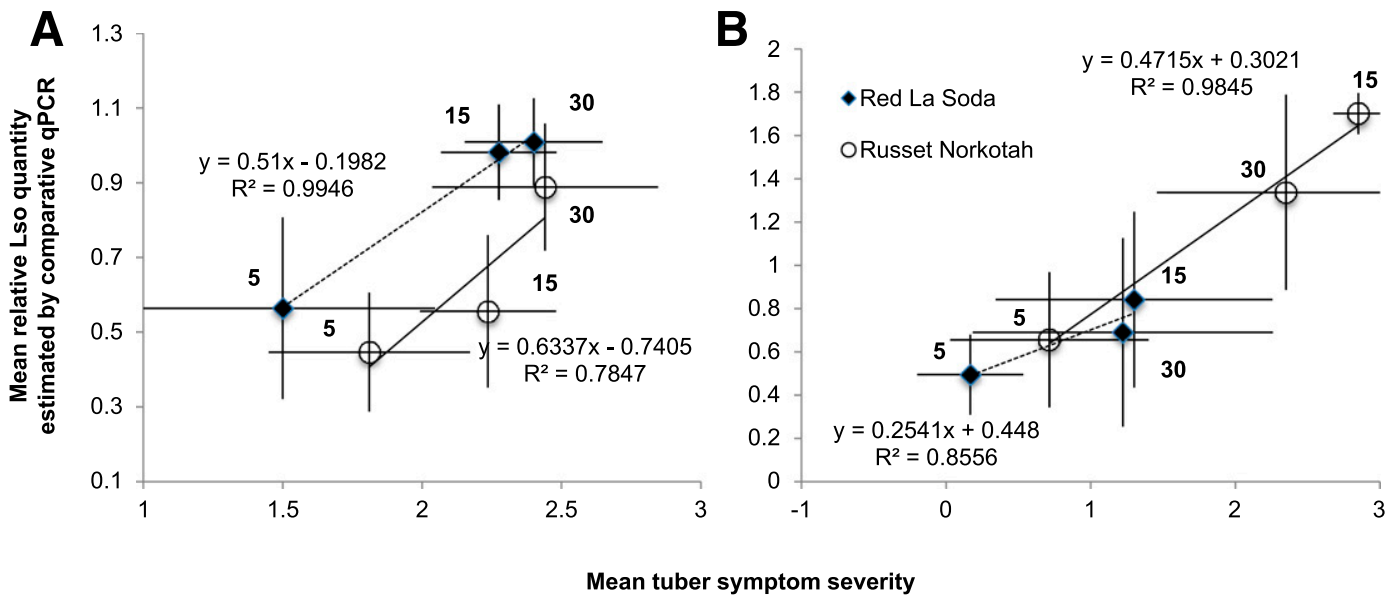

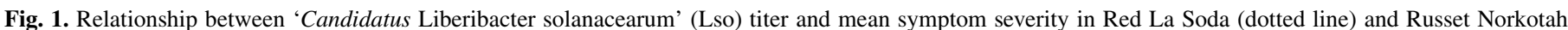
(solid line) in A, 2012 and B, 2013. Error bars reflect 95\% confidence intervals.

TABLE 1. Mean amino acid concentrations for each of the psyllid density treatments in Red La Soda and Russet Norkotah

\begin{tabular}{|c|c|c|c|c|c|c|c|c|}
\hline \multirow[b]{3}{*}{ Cultivar, amino acid } & \multicolumn{8}{|c|}{ Concentration $\left(\mu \mathrm{mol} / \mathrm{g}\right.$ fresh weight \pm standard error) per year and psyllid density ${ }^{z}$} \\
\hline & \multicolumn{4}{|c|}{2012} & \multicolumn{4}{|c|}{2013} \\
\hline & 0 & 5 & 15 & 30 & 0 & 5 & 15 & 30 \\
\hline \multicolumn{9}{|l|}{ Red La Soda } \\
\hline Asparagine & $23.12 \pm 4.3 \mathrm{a}$ & $12.38 \pm 2.58 \mathrm{a}$ & $12.035 \pm 2.54 \mathrm{a}$ & $11.29 \pm 2.45 \mathrm{a}$ & $26.96 \pm 2.04 \mathrm{a}$ & $29.49 \pm 2.44 \mathrm{a}$ & $28.03 \pm 2.75 \mathrm{a}$ & $35.38 \pm 6.61 \mathrm{a}$ \\
\hline Aspartic acid & $14.35 \pm 1.46 \mathrm{a}$ & $12.85 \pm 1.56 \mathrm{a}$ & $10.61 \pm 0.94 \mathrm{a}$ & $9.70 \pm 1.13 \mathrm{a}$ & $2.30 \pm 0.10 \mathrm{a}$ & $2.89 \pm 2.31 \mathrm{a}$ & $2.62 \pm 0.24 \mathrm{a}$ & $2.96 \pm 0.54 \mathrm{a}$ \\
\hline Glutamic acid & $2.78 \pm 0.39 a$ & $3.07 \pm 0.347 \mathrm{a}$ & $2.08 \pm 0.258 \mathrm{a}$ & $1.92 \pm 0.32 \mathrm{a}$ & $1.28 \pm 0.13 \mathrm{a}$ & $1.19 \pm 0.14 \mathrm{ab}$ & $0.92 \pm 0.13 a b$ & $0.73 \pm 0.15 b$ \\
\hline Glutamine & $7.46 \pm 1.52 \mathrm{a}$ & $5.73 \pm 1.24 \mathrm{a}$ & $5.10 \pm 1.073 \mathrm{a}$ & $5.56 \pm 1.33 \mathrm{a}$ & $7.35 \pm 0.65 \mathrm{a}$ & $7.93 \pm 0.90 \mathrm{a}$ & $8.57 \pm 0.99 \mathrm{a}$ & $6.75 \pm 1.21 \mathrm{a}$ \\
\hline Histidine & $0.68 \pm 0.55 \mathrm{a}$ & $0.83 \pm 0.117 \mathrm{a}$ & $0.88 \pm 0.08 \mathrm{a}$ & $0.91 \pm 0.075 \mathrm{a}$ & $0.65 \pm 0.05 \mathrm{a}$ & $0.71 \pm 0.07 \mathrm{a}$ & $1.03 \pm 0.20 \mathrm{a}$ & $1.00 \pm 0.18 \mathrm{a}$ \\
\hline Isoleucine & $0.90 \pm 0.10 \mathrm{a}$ & $2.12 \pm 0.261 b$ & $2.59 \pm 0.199 \mathrm{~b}$ & $2.49 \pm 0.136 b$ & $1.12 \pm 0.12 \mathrm{a}$ & $1.12 \pm 0.14 \mathrm{a}$ & $1.86 \pm 0.25 \mathrm{a}$ & $1.57 \pm 0.28 \mathrm{a}$ \\
\hline Leucine & $0.57 \pm 0.17 \mathrm{a}$ & $1.19 \pm 0.13 \mathrm{~b}$ & $1.44 \pm 0.097 \mathrm{~b}$ & $1.44 \pm 0.098 b$ & $0.58 \pm 0.09 \mathrm{a}$ & $0.65 \pm 0.12 \mathrm{a}$ & $1.09 \pm 0.16 \mathrm{a}$ & $0.77 \pm 0.13 \mathrm{a}$ \\
\hline Lysine & $0.99 \pm 0.103 \mathrm{a}$ & $1.63 \pm 0.213 \mathrm{ab}$ & $1.60 \pm 0.147 \mathrm{ab}$ & $1.75 \pm 0.162 b$ & $0.549 \pm 0.05 \mathrm{a}$ & $0.62 \pm 0.07 \mathrm{a}$ & $0.84 \pm 0.08 \mathrm{a}$ & $0.63 \pm 0.05 \mathrm{a}$ \\
\hline Methionine & $1.13 \pm 0.064 \mathrm{a}$ & $1.11 \pm 0.057 \mathrm{a}$ & $1.08 \pm 0.055 \mathrm{a}$ & $1.02 \pm 0.061 \mathrm{a}$ & $0.58 \pm 0.05 \mathrm{a}$ & $0.52 \pm 0.06 \mathrm{ab}$ & $0.35 \pm 0.051 \mathrm{ab}$ & $0.15 \pm 0.01 \mathrm{~b}$ \\
\hline Phenylalanine & $0.86 \pm 0.07 \mathrm{a}$ & $1.50 \pm 0.145 b$ & $1.78 \pm 0.141 \mathrm{~b}$ & $1.79 \pm 0.114 b$ & $1.08 \pm 0.09 \mathrm{a}$ & $1.02 \pm 0.15 \mathrm{a}$ & $1.23 \pm 0.17 \mathrm{a}$ & $0.77 \pm 0.11 \mathrm{a}$ \\
\hline Proline & $0.61 \pm 0.12 \mathrm{a}$ & $2.23 \pm 0.365 b$ & $2.98 \pm 0.261 \mathrm{~b}$ & $2.49 \pm 0.211 b$ & $1.99 \pm 0.35 \mathrm{a}$ & $1.60 \pm 0.39 \mathrm{a}$ & $2.21 \pm 0.26 \mathrm{a}$ & $1.35 \pm 0.30 \mathrm{a}$ \\
\hline Serine & $14.09 \pm 0.11 \mathrm{a}$ & $18.35 \pm 2.23 \mathrm{a}$ & $18.99 \pm 1.39 \mathrm{a}$ & $18.12 \pm 1.21 \mathrm{a}$ & $5.63 \pm 0.29 \mathrm{a}$ & $5.99 \pm 0.36 \mathrm{a}$ & $6.26 \pm 0.54 \mathrm{a}$ & $5.48 \pm 0.65 \mathrm{a}$ \\
\hline Tryptophan & $0.57 \pm 0.018 \mathrm{a}$ & $0.77 \pm 0.084 \mathrm{a}$ & $0.77 \pm 0.068 \mathrm{a}$ & $0.78 \pm 0.053 \mathrm{a}$ & $0.24 \pm 0.02 \mathrm{a}$ & $0.24 \pm 0.02 \mathrm{ab}$ & $0.54 \pm 0.08 \mathrm{c}$ & $0.38 \pm 0.07 a b c$ \\
\hline Valine & $4.81 \pm 0.54 \mathrm{a}$ & $10.28 \pm 0.918 b$ & $10.29 \pm 0.476 b$ & $9.90 \pm 0.064 b$ & $3.27 \pm 0.26 \mathrm{a}$ & $3.04 \pm 0.37 \mathrm{a}$ & $4.19 \pm 0.34 \mathrm{a}$ & $3.17 \pm 0.43 \mathrm{a}$ \\
\hline \multicolumn{9}{|l|}{ Russet Norkotah } \\
\hline Asparagine & $13.16 \pm 2.56 \mathrm{a}$ & $12.21 \pm 2.02 \mathrm{a}$ & $16.94 \pm 2.08 \mathrm{a}$ & $13.37 \pm 1.66 \mathrm{a}$ & $6.73 \pm 1.16 \mathrm{a}$ & $4.65 \pm 0.83 \mathrm{a}$ & $7.82 \pm 0.89 a b$ & $13.02 \pm 2.05 \mathrm{~b}$ \\
\hline Aspartic acid & $9.97 \pm 1.05 \mathrm{a}$ & $9.13 \pm 1.16 \mathrm{a}$ & $9.86 \pm 1.12 \mathrm{a}$ & $9.59 \pm 1.24 \mathrm{a}$ & $1.47 \pm 0.15 \mathrm{a}$ & $1.75 \pm 0.27 \mathrm{a}$ & $2.04 \pm 0.16 \mathrm{a}$ & $2.19 \pm 0.22 \mathrm{a}$ \\
\hline Glutamic acid & $1.88 \pm 0.21 \mathrm{a}$ & $1.63 \pm 0.234 \mathrm{a}$ & $1.72 \pm 0.28 \mathrm{a}$ & $2.32 \pm 0.37 \mathrm{a}$ & $0.42 \pm 0.04 \mathrm{a}$ & $0.49 \pm 0.09 \mathrm{a}$ & $0.22 \pm 0.02 \mathrm{a}$ & $0.39 \pm 0.08 \mathrm{a}$ \\
\hline Glutamine & $4.73 \pm 1.11 \mathrm{a}$ & $9.32 \pm 1.66 \mathrm{ab}$ & $14.59 \pm 1.91 \mathrm{~b}$ & $14.05 \pm 2.43 \mathrm{~b}$ & $5.04 \pm 0.78 \mathrm{ab}$ & $3.32 \pm 0.61 \mathrm{a}$ & $9.38 \pm 0.76 b$ & $10.14 \pm 0.77 \mathrm{bc}$ \\
\hline Histidine & $0.50 \pm 0.04 \mathrm{a}$ & $1.33 \pm 0.197 \mathrm{~b}$ & $1.62 \pm 0.19 b$ & $1.82 \pm 0.28 b$ & $0.17 \pm 0.02 \mathrm{a}$ & $0.17 \pm 0.02 \mathrm{a}$ & $0.65 \pm 0.08 \mathrm{~b}$ & $0.68 \pm 0.07 \mathrm{~b}$ \\
\hline Isoleucine & $0.61 \pm 0.052 \mathrm{a}$ & $2.57 \pm 0.3 \mathrm{~b}$ & $3.01 \pm 0.3 b$ & $3.13 \pm 0.34 \mathrm{~b}$ & $0.23 \pm 0.05 \mathrm{a}$ & $0.23 \pm 0.03 \mathrm{a}$ & $1.25 \pm 0.14 \mathrm{~b}$ & $1.34 \pm 0.16 b$ \\
\hline Leucine & $0.48 \pm 0.05 \mathrm{a}$ & $1.88 \pm 0.22 b$ & $1.95 \pm 0.16 b$ & $1.90 \pm 0.19 b$ & $0.099 \pm 0.02 \mathrm{a}$ & $0.19 \pm 0.05 \mathrm{a}$ & $1.02 \pm 0.11 \mathrm{~b}$ & $1.05 \pm 0.14 \mathrm{~b}$ \\
\hline Lysine & $0.72 \pm 0.08 \mathrm{a}$ & $1.74 \pm 0.228 \mathrm{~b}$ & $2.32 \pm 0.28 \mathrm{~b}$ & $2.44 \pm 0.37 \mathrm{~b}$ & $0.21 \pm 0.05 \mathrm{a}$ & $0.23 \pm 0.02 \mathrm{a}$ & $0.65 \pm 0.07 \mathrm{~b}$ & $0.62 \pm 0.05 \mathrm{~b}$ \\
\hline Methionine & $0.80 \pm 0.05 \mathrm{a}$ & $0.80 \pm 0.066 \mathrm{a}$ & $0.93 \pm 0.06 \mathrm{a}$ & $0.95 \pm 0.06 \mathrm{a}$ & $0.10 \pm 0.02 \mathrm{a}$ & $0.07 \pm 0.01 \mathrm{a}$ & $0.20 \pm 0.02 b$ & $0.18 \pm 0.01 \mathrm{~b}$ \\
\hline Phenylalanine & $0.59 \pm 0.04 \mathrm{a}$ & $1.30 \pm 0.13 b$ & $1.63 \pm 0.14 \mathrm{~b}$ & $1.58 \pm 0.13 b$ & $0.19 \pm 0.03 \mathrm{a}$ & $0.21 \pm 0.02 \mathrm{a}$ & $0.55 \pm 0.06 \mathrm{~b}$ & $0.64 \pm 0.08 \mathrm{~b}$ \\
\hline Proline & $0.34 \pm 0.05 \mathrm{a}$ & $2.41 \pm 0.33 b$ & $2.92 \pm 0.31 \mathrm{~b}$ & $2.75 \pm 0.32 b$ & $0.21 \pm 0.04 \mathrm{a}$ & $0.25 \pm 0.04 \mathrm{a}$ & $1.51 \pm 0.17 b$ & $1.56 \pm 0.20 \mathrm{~b}$ \\
\hline Serine & $16.33 \pm 1.48 \mathrm{a}$ & $20.59 \pm 1.52 \mathrm{a}$ & $20.77 \pm 1.76 \mathrm{a}$ & $19.34 \pm 2.32 \mathrm{a}$ & $2.54 \pm 0.32 \mathrm{a}$ & $4.04 \pm 0.36 \mathrm{ab}$ & $5.87 \pm 0.48 b$ & $5.71 \pm 0.47 \mathrm{~b}$ \\
\hline Tryptophan & $0.53 \pm 0.02 \mathrm{a}$ & $1.14 \pm 0.136 \mathrm{~b}$ & $1.35 \pm 0.16 b$ & $1.62 \pm 0.26 \mathrm{~b}$ & $0.091 \pm 0.03 \mathrm{a}$ & $0.10 \pm 0.02 \mathrm{a}$ & $0.46 \pm 0.05 \mathrm{~b}$ & $0.47 \pm 0.06 \mathrm{~b}$ \\
\hline Valine & $3.34 \pm 0.27 \mathrm{a}$ & $8.65 \pm 1.16 \mathrm{ab}$ & $11.20 \pm 1.3 \mathrm{~b}$ & $11.05 \pm 1.27 \mathrm{~b}$ & $0.86 \pm 0.18 \mathrm{a}$ & $0.89 \pm 0.12 \mathrm{a}$ & $2.79 \pm 0.26 \mathrm{~b}$ & $3.03 \pm 0.31 \mathrm{~b}$ \\
\hline
\end{tabular}

${ }^{\mathrm{z}}$ Different letters indicate significant differences $(P<0.05)$ across density treatments. 
greatly positively correlated (all $P$ values $<0.005$ ) (Table 2 ) with ' $C a$. L. solanacearum' quantity within tuber tissue.

In Russet Norkotah, aspartic acid $(\rho=0.066, n=90, P=0.535)$ and glutamic acid $(\rho=-0.011, n=90, P=0.918)$ levels were not associated with tuber symptoms. Levels of all remaining amino acids were significantly positively affected by the degree of symptom severity (all $P$ values $<0.038$ ).

In Russet Norkotah, levels of serine (Pearson corr. $=-0.261, n=87$, $P=0.015)$, aspartic acid (Pearson corr. $=-0.369, n=88, P<0.001$ ), methionine (Pearson corr. $=-0.256, n=88, P=0.016$ ), glutamic acid (Pearson corr. $=-0.351, n=88, P=0.001$ ) and glutamine (Pearson corr. $=0.225, n=88, P=0.035$ ) were significantly associated with 'Ca. L. solanacearum' titer (Table 2).

Phenolics. Significant psyllid density-cultivar (Pillai's Trace $=$ $0.538, F_{33,606}=4.14, P<0.001$ ) and year-cultivar (Pillai's Trace $=$ $\left.0.586, F_{11,200}=25.72, P<0.001\right)$ interactions were detected when MANOVA of phenolics was performed; therefore, subsequent analyses of the phenolic compounds were conducted independently for each of the two cultivars.

In Red La Soda, levels of all evaluated phenolic compounds were affected by the psyllid density treatments (all $P$ values $<0.001$ ), with the exception of gallic acid $\left(F_{3,106}=1.19, P=0.318\right)$. Phenolic levels were significantly lower in tubers from noninfested plants than the infected treatments. Moreover, lower phenolic levels were detected in plants that were infested with 5 psyllids compared with those infested with 15 and 30 infective psyllids (Table 3).
In Russet Norkotah, levels of all 11 phenolic compounds were significantly affected by the psyllid density treatments (all $P$ values $<0.001$ ) (Table 3 ). With the exception of gallic acid, noninfested control plants generally had significantly lower phenolic levels than the infested treatments (Table 3). Likewise, plants infested with 5 infective psyllids showed lower levels in 8 of the 11 evaluated phenolics when compared with 15- and 30-psyllid treatments.

In Red La Soda, levels of all phenolic compounds exhibited a significant positive correlation with ' $\mathrm{Ca}$. L. solanacearum' titer (Pearson corr., all $P$ values $<00.008$ ), with the exception of gallic acid (Pearson corr. $=-0.036, n=83, P=0.744$ ) (Table 4).

There was a negative association between symptom severity and gallic acid levels in Red La Soda tubers $(\rho=-0.344, n=83, P=0.001)$. All remaining phenolic levels were highly positively correlated with the degree of symptom severity in tuber tissue (all $P$ values $<00.002$ ).

In Russet Norkotah, levels of two of the flavonoid glycoside derivatives and one of the cryptochlorogenic acid derivatives were the only compounds that were not associated with ' $\mathrm{Ca}$. L. solanacearum' titers (all $P$ values $>0.118$ ). Levels of the other phenolics had positive correlations with ' $\mathrm{Ca}$. L. solanacearum' titers (Pearson corr., all $P$ values $<0.013$ ) (Table 4). Only gallic acid levels were not affected by increase in symptom severity $(\rho=-0.152, n=78, P=0.185)$. All remaining phenolic levels were positively correlated with tuber symptoms (all $P$ values $<0.001$ ).

Carbohydrates. Similar to amino acid and phenolic analyses, cultivars were analyzed separately as significant year-cultivar

TABLE 2. Relationship between 'Candidatus Liberibacter solanacearum' titer and amino acid concentrations in Red La Soda and Russet Norkotah

\begin{tabular}{|c|c|c|c|c|c|c|c|c|c|c|c|c|c|c|}
\hline \multirow[b]{2}{*}{ Cultivar $^{2}$} & \multicolumn{14}{|c|}{ Amino $\operatorname{acids}^{y}$} \\
\hline & Asparagine & Aspartic acid & Glutamic acid & Glutamine & Histidine & Isoleucine & Leucine & Lysine & Methionine & Phenylalanine & Proline & Serine & Tryptophan & Valine \\
\hline \multicolumn{15}{|c|}{ Red La Soda } \\
\hline Pearson & 0.052 & 0.166 & -0.148 & 0.104 & $0.461 * *$ & $0.559 * *$ & $0.531 * *$ & $0.389 * *$ & 0.146 & $0.446 * *$ & $0.366 * *$ & $0.399 * *$ & $0.523 * *$ & $0.305^{* *}$ \\
\hline$P$ value & 0.629 & 0.124 & 0.172 & 0.338 & $<0.001$ & $<0.001$ & $<0.001$ & $<0.001$ & 0.178 & $<0.001$ & $<0.002$ & $<0.003$ & $<0.004$ & 0.004 \\
\hline$N$ & 87 & 87 & 87 & 87 & 86 & 87 & 87 & 86 & 87 & 87 & 87 & 87 & 85 & 87 \\
\hline \multicolumn{15}{|c|}{ Russet Norkotah } \\
\hline Pearson & 0.038 & $-0.369 * *$ & $-0.351 * *$ & $0.225 *$ & -0.033 & 0.048 & 0.072 & -0.135 & $-0.256^{*}$ & -0.053 & 0.144 & $-0.261 *$ & -0.076 & -0.04 \\
\hline$P$ value & 0.724 & $<0.001$ & $<0.001$ & 0.035 & 0.759 & 0.656 & 0.503 & 0.210 & 0.016 & 0.625 & 0.185 & 0.015 & 0.487 & 0.713 \\
\hline$N$ & 88 & 88 & 88 & 88 & 87 & 88 & 88 & 88 & 88 & 88 & 87 & 87 & 87 & 88 \\
\hline
\end{tabular}

y Significance: $* *$ and $*$ indicate that correlation is significant at the 0.01 and 0.05 level, respectively.

${ }^{\mathrm{z}}$ Pearson $=$ Pearson correlation

TABLE 3. Mean concentrations of the phenolic compounds for each of the psyllid density treatments in Red La Soda and Russet Norkotah

\begin{tabular}{|c|c|c|c|c|c|c|c|c|}
\hline \multirow[b]{3}{*}{ Cultivar, compound $^{2}$} & \multicolumn{8}{|c|}{ Concentration ( $\mu \mathrm{g} / \mathrm{g}$ fresh weight \pm standard error) per year and psyllid density ${ }^{\mathrm{y}}$} \\
\hline & \multicolumn{4}{|c|}{2012} & \multicolumn{4}{|c|}{2013} \\
\hline & 0 & 5 & 15 & 30 & 0 & 5 & 15 & 30 \\
\hline \multicolumn{9}{|l|}{ Red La Soda } \\
\hline Chlorogenic acid 1 & $2.16 \pm 0.92 \mathrm{a}$ & $204.9 \pm 52.2 \mathrm{~b}$ & $304.09 \pm 36.62 \mathrm{bc}$ & $320.74 \pm 30.89 \mathrm{c}$ & $3.68 \pm 0.50 \mathrm{a}$ & $41.91 \pm 37.3 \mathrm{ab}$ & $71.38 \pm 24.74 \mathrm{~b}$ & $33.45 \pm 10.72 \mathrm{ab}$ \\
\hline Chlorogenic acid 2 & $10.6 \pm 2.63 \mathrm{a}$ & $177.4 \pm 37.8 \mathrm{~b}$ & $328.85 \pm 40.17 \mathrm{~b}$ & $363.05 \pm 34.83 \mathrm{~b}$ & $2.17 \pm 0.26 \mathrm{a}$ & $17.55 \pm 14.61 \mathrm{ab}$ & $125.87 \pm 55.78 b$ & $24.64 \pm 15.24 \mathrm{ab}$ \\
\hline Crypto-c acid 1 & $5.43 \pm 0.64 \mathrm{a}$ & $78.01 \pm 17.5 \mathrm{~b}$ & $137.13 \pm 15.20 \mathrm{c}$ & $160.11 \pm 12.50 \mathrm{c}$ & $0.16 \pm 0.05 \mathrm{a}$ & $2.15 \pm 1.85 \mathrm{a}$ & $20.96 \pm 12.21 \mathrm{a}$ & $1.12 \pm 0.34 \mathrm{a}$ \\
\hline Crypto-c acid 2 & $1.21 \pm 0.83 \mathrm{a}$ & $104.5 \pm 22.5 \mathrm{~b}$ & $186.21 \pm 19.52 \mathrm{c}$ & $260.07 \pm 14.97 \mathrm{c}$ & $0.66 \pm 0.09 \mathrm{a}$ & $1.67 \pm 1.0 \mathrm{ab}$ & $62.17 \pm 24.063 \mathrm{~b}$ & $10.78 \pm 4.35 \mathrm{ab}$ \\
\hline Flav glyc 1 & $16.8 \pm 2.10 \mathrm{a}$ & $70.73 \pm 8.99 \mathrm{~b}$ & $99.94 \pm 5.63 \mathrm{~b}$ & $106.96 \pm 4.42 b$ & $16.28 \pm 1.92 \mathrm{a}$ & $12.38 \pm 1.63 \mathrm{a}$ & $22.38 \pm 5.63 \mathrm{a}$ & $16.22 \pm 3.42 \mathrm{a}$ \\
\hline Flav glyc 2 & $7.27 \pm 1.10 \mathrm{a}$ & $69.95 \pm 12.0 \mathrm{~b}$ & $100.97 \pm 10.37 \mathrm{bc}$ & $128.82 \pm 8.69 \mathrm{c}$ & $6.32 \pm 1.16 \mathrm{a}$ & $5.9 \pm 1.70 \mathrm{a}$ & $18.85 \pm 6.04 \mathrm{a}$ & $4.39 \pm 1.51 \mathrm{a}$ \\
\hline Flav glyc 3 & $2.76 \pm 0.65 \mathrm{a}$ & $39.72 \pm 7.03 b$ & $62.25 \pm 6.45 \mathrm{bc}$ & $76.72 \pm 4.84 \mathrm{c}$ & $0.09 \pm 0.05 \mathrm{a}$ & $0.26 \pm 0.18 \mathrm{a}$ & $4.61 \pm 4.01 \mathrm{a}$ & $1.47 \pm 0.83 \mathrm{a}$ \\
\hline Gallic acid & $10.1 \pm 0.57 \mathrm{a}$ & $12.81 \pm 0.58 \mathrm{~b}$ & $13.01 \pm 0.30 \mathrm{~b}$ & $12.05 \pm 0.47 \mathrm{ab}$ & $39.9 \pm 2.54 \mathrm{a}$ & $31.57 \pm 2.17 \mathrm{a}$ & $36.77 \pm 3.50 \mathrm{a}$ & $38.48 \pm 5.94 \mathrm{a}$ \\
\hline PA hexoside & $17.8 \pm 2.12 \mathrm{a}$ & $59.55 \pm 5.90 \mathrm{~b}$ & $80.5 \pm 3.83 b$ & $83.12 \pm 2.08 \mathrm{~b}$ & $71.74 \pm 9.61 \mathrm{a}$ & $60.96 \pm 7.44 \mathrm{a}$ & $120.63 \pm 27.84 \mathrm{a}$ & $108.46 \pm 25.71 \mathrm{a}$ \\
\hline Quinic acid & $5.04 \pm 0.60 \mathrm{a}$ & $17.56 \pm 1.51 \mathrm{~b}$ & $21.58 \pm 1.19 \mathrm{~b}$ & $23.93 \pm 1.00 \mathrm{~b}$ & $25.84 \pm 1.41 \mathrm{a}$ & $24.63 \pm 1.77 \mathrm{a}$ & $34.685 \pm 4.86 \mathrm{a}$ & $43.84 \pm 6.64 \mathrm{~b}$ \\
\hline Salicylic acid & $0.12 \pm 0.03 \mathrm{a}$ & $6.95 \pm 1.49 \mathrm{~b}$ & $11.50 \pm 1.23 \mathrm{c}$ & $13.42 \pm 0.75 \mathrm{c}$ & $1.06 \pm 0.30 \mathrm{a}$ & $2.65 \pm 1.35 \mathrm{a}$ & $12.57 \pm 5.43 \mathrm{a}$ & $8.18 \pm 3.42 \mathrm{a}$ \\
\hline \multicolumn{9}{|l|}{ Russet Norkotah } \\
\hline Chlorogenic acid 1 & $1.76 \pm 0.67 \mathrm{a}$ & $442.44 \pm 65.15 b$ & $310.67 \pm 49.98 b$ & $340.73 \pm 48.05 b$ & $10.83 \pm 2.06 \mathrm{a}$ & $111.82 \pm 68.81 \mathrm{a}$ & $422.36 \pm 90.83 b$ & $202.79 \pm 40.62 \mathrm{ab}$ \\
\hline Chlorogenic acid 2 & $11.24 \pm 2.36 \mathrm{a}$ & $548.82 \pm 53.14 b$ & $334.55 \pm 62.73 \mathrm{bc}$ & $422.7 \pm 41.45 \mathrm{c}$ & $27.88 \pm 5.63 \mathrm{a}$ & $123.68 \pm 64.42 \mathrm{a}$ & $514.95 \pm 63.15 b$ & $485.38 \pm 89.35 b$ \\
\hline Crypto-c acid 1 & $6.76 \pm 0.45 \mathrm{a}$ & $151.57 \pm 14.90 b$ & $114.82 \pm 18.96 \mathrm{~b}$ & $142.07 \pm 11.59 \mathrm{~b}$ & $8.26 \pm 1.80 \mathrm{a}$ & $25.05 \pm 12.89 \mathrm{a}$ & $207.57 \pm 32.15 b$ & $144.35 \pm 32.08 b$ \\
\hline Crypto-c acid 2 & $2.57 \pm 0.41 \mathrm{a}$ & $275.9 \pm 26.89 \mathrm{~b}$ & $232.21 \pm 32.12 \mathrm{~b}$ & $309.71 \pm 19.37 \mathrm{~b}$ & $3.99 \pm 0.76 \mathrm{a}$ & $33.05 \pm 22.53 \mathrm{a}$ & $171.13 \pm 31.21 \mathrm{~b}$ & $140.17 \pm 30.66 b$ \\
\hline Flav glyc 1 & $9.97 \pm 1.88 \mathrm{a}$ & $114.9 \pm 8.54 \mathrm{~b}$ & $99.5 \pm 10.09 \mathrm{~b}$ & $116.69 \pm 5.96 b$ & $3.26 \pm 1.50 \mathrm{a}$ & $5.11 \pm 1.10 \mathrm{a}$ & $51.07 \pm 5.09 \mathrm{~b}$ & $35.09 \pm 8.47 \mathrm{~b}$ \\
\hline Flav glyc 2 & $2.46 \pm 0.43 \mathrm{a}$ & $117.36 \pm 8.46 b$ & $89.65 \pm 13.03 b$ & $87.9 \pm 5.92 b$ & $1.47 \pm 0.69 \mathrm{a}$ & $3.60 \pm 2.81 \mathrm{a}$ & $31.08 \pm 2.44 \mathrm{~b}$ & $25.81 \pm 5.46 \mathrm{~b}$ \\
\hline Flav glyc 3 & $0.56 \pm 0.18 \mathrm{a}$ & $69.64 \pm 5.89 \mathrm{~b}$ & $44.19 \pm 8.11 \mathrm{bc}$ & $40.62 \pm 9.37 \mathrm{c}$ & $1.03 \pm 0.28 \mathrm{a}$ & $13.03 \pm 7.46 \mathrm{a}$ & $120.31 \pm 17.89 b$ & $70.15 \pm 16.56 \mathrm{~b}$ \\
\hline Gallic acid & $10.67 \pm 0.43 a$ & $10.35 \pm 0.29 a$ & $9.67 \pm 0.41 \mathrm{a}$ & $9.25 \pm 0.43 \mathrm{a}$ & $29.77 \pm 3.11 \mathrm{a}$ & $29.83 \pm 2.03 \mathrm{a}$ & $28.85 \pm 2.12 \mathrm{a}$ & $22.43 \pm 1.95 \mathrm{a}$ \\
\hline PA hexoside & $11.47 \pm 2.01 \mathrm{a}$ & $74.88 \pm 3.36 b$ & $72.21 \pm 6.78 b$ & $80.75 \pm 3.87 b$ & $38.19 \pm 14.01 \mathrm{a}$ & $20.56 \pm 5.50 \mathrm{a}$ & $196.43 \pm 7.50 \mathrm{~b}$ & $161.74 \pm 18.92 b$ \\
\hline Quinic acid & $3.38 \pm 0.37 \mathrm{a}$ & $15.56 \pm 0.81 \mathrm{~b}$ & $15.39 \pm 1.24 \mathrm{~b}$ & $17.3 \pm 0.82 b$ & $17.57 \pm 2.41 \mathrm{a}$ & $18.62 \pm 2.27 \mathrm{a}$ & $45.73 \pm 2.37 \mathrm{~b}$ & $38.98 \pm 3.71 \mathrm{~b}$ \\
\hline Salicylic acid & $0.17 \pm 0.01 \mathrm{a}$ & $18.37 \pm 2.22 b$ & $13.61 \pm 1.89 \mathrm{~b}$ & $17.17 \pm 1.98 \mathrm{~b}$ & $0.96 \pm 0.27 \mathrm{a}$ & $10.06 \pm 5.21 \mathrm{a}$ & $50.87 \pm 4.77 \mathrm{~b}$ & $36.02 \pm 8.22 b$ \\
\hline
\end{tabular}

y Different letters indicate significant differences $(P<0.05)$ across density treatments.

${ }^{\mathrm{z}}$ Crypto-c $=$ cryptochlorogenic acid, Flav glyc $=$ flavonoid glycoside, and PA hexoside $=$ protocatechuic acid hexoside. 
(Pillai's Trace $=0.337, F_{3,225}=38.18, P<0.001$ ) and psyllid density-cultivar (Pillai's Trace $=0.162, F_{9,681}=4.31, P<0.001$ ) interactions were detected.

In Red La Soda, significant effects of psyllid density (Pillai's Trace $\left.=0.370, F_{9,339}=5.30, P<0.001\right)$, year (Pillai's Trace $=$ $0.520, F_{3,111}=40.12, P<0.001$ ), and plant replicate (Pillai's Trace $\left.=0.380, F_{12,339}=4.10, P<0.001\right)$ were detected; thus, these variables remained in the final model. Noninfested control plants had significantly lower concentrations of glucose than the plants infested with either 15 or 30 psyllids (all $P$ values $<0.001$ ), regardless of the treatment. Similarly for fructose, noninfested plants and plants infested with 5 infective psyllids formed a homogenous subset $(P=$ 00.201), which was significantly different from plants infested with 15 and 30 psyllids (all $P$ values $<0.001$ ).

In Russet Norkotah, sugar concentrations showed variations across years (Pillai's Trace $=0.284, F_{3,108}=14.31, P<0.001$ ) and psyllid density (Pillai's Trace $=0.372, F_{9,330}=5.19, P<0.001$ ) but not for plant replicate (Pillai's Trace $=0.182, F_{12,330}=1.77, P=0.052$ ) .

Although fructose concentrations were significantly lower in the noninfested controls than the infested treatments, glucose concentrations showed a different and inconsistent pattern. Glucose concentrations in the noninfested control formed a homogenous statistical subset $(P=0.061)$ with 5- and 30-psyllid treatments, and were only statistically different from plants infested with 15 infective psyllids $(P=0.016)$ (Table 5).

In Red La Soda, elevated glucose (Pearson corr. $=0.425, n=87$, $P<0.001)$ and fructose (Pearson corr. $=0.459, n=87, P<0.001$ ) levels were associated with increased ' $\mathrm{Ca}$. L. solanacearum' titer. Sucrose concentration was not influenced by ' $\mathrm{C} a$. L. solanacearum' titer (Pearson corr. $=-0.138, n=87, P=0.202$ ) (Table 6).

In Red La Soda, the degree of symptom severity was positively correlated with glucose $(\rho=0.520, n=88, P<0.001)$ and fructose ( $\rho=0.352, n=88, P=0.001)$ levels and negatively correlated with sucrose $(\rho=-0.401, n=88, P<0.001)$.
In Russet Norkotah, fructose was the only sugar whose levels were affected by change in ' $\mathrm{Ca}$. L. solanacearum' titer (Pearson corr. $=0.388, n=86, P<0.001)($ Table 6$)$. Likewise, only fructose levels were positively associated with the severity of ZC symptoms in tuber tissue (Pearson corr. $=0.533, n=88, P<0.001$ ).

\section{DISCUSSION}

This study was conducted to evaluate whether or not shifts in tuber physiology that are triggered by ' $\mathrm{Ca}$. L. solanacearum' infection and that are associated with ZC symptom expression can be influenced by variations in vector density. Results showed that, in Red La Soda and Russet Norkotah potato, ' $\mathrm{Ca}$. L. solanacearum' titer and the severity of the tuber symptoms, as well as some biochemical properties associated with symptom expression, varied among vector density treatments. In some cases, significant effects of year or year-cultivar interaction were detected. In relation to this, it is known that the development of Liberibacter spp. is influenced by environmental factors (Bove 2006; Lopes et al. 2009; Munyaneza et al. 2012). If a significant year-cultivar interaction was detected, results were presented independently.

Although vector titer by itself does not appear to directly influence ' $C a$. L. solanacearum' levels in the infected plant tissue, increase in the number of bacteriliferous psyllids is shown to result in elevated in ' $\mathrm{Ca}$. L. solanacearum' titer in foliar tissue days after inoculation (Rashed et al. 2012). This was also supported by an earlier study by Buchman and colleagues (2011), who concluded that the likelihood of detecting ' $\mathrm{Ca}$. L. solanacearum' in potato ('Atlantic') infested with a single psyllid was lower than in plants infested with 20 psyllids, due to low titer of the bacterium. Thus, the observed variations in tuber ' $C a$. L. solanacearum' titer and symptom severity in relation to vector density in the present study may be due to differences in the initial ' $C a$. L. solanacearum' inoculum (titer) level injected into the plant tissue. Variations in ' $\mathrm{Ca}$. L. solanacearum' concentrations may

TABLE 4. Relationships between 'Candidatus Liberibacter solanacearum' titer and evaluated phenolic compounds

\begin{tabular}{|c|c|c|c|c|c|c|c|c|c|c|c|}
\hline \multirow[b]{2}{*}{ Cultivar $^{z}$} & \multicolumn{11}{|c|}{ Phenolic compounds ${ }^{y}$} \\
\hline & $\begin{array}{l}\text { Chlorogenic } \\
\text { acid } 1\end{array}$ & $\begin{array}{l}\text { Chlorogenic } \\
\text { acid } 2\end{array}$ & $\begin{array}{l}\text { Crypto-c } \\
\text { acid } 1\end{array}$ & $\begin{array}{l}\text { Crypto-c } \\
\text { acid } 2\end{array}$ & $\begin{array}{l}\text { Flav } \\
\text { glyc } 1\end{array}$ & $\begin{array}{l}\text { Flav } \\
\text { glyc } 2\end{array}$ & $\begin{array}{l}\text { Flav } \\
\text { glyc } 3\end{array}$ & $\begin{array}{l}\text { Gallic } \\
\text { acid }\end{array}$ & $\begin{array}{c}\text { PA } \\
\text { hexoside }\end{array}$ & $\begin{array}{l}\text { Quinic } \\
\text { acid }\end{array}$ & $\begin{array}{c}\text { Salicylic } \\
\text { acid }\end{array}$ \\
\hline \multicolumn{12}{|c|}{ Red La Soda } \\
\hline Pearson & $0.683 * *$ & $0.636^{* *}$ & $0.537 * *$ & $0.682 * *$ & $0.583^{* *}$ & $0.610 * *$ & $0.504 * *$ & -0.036 & $0.735 * *$ & $0.419 * *$ & $0.832 * *$ \\
\hline$P$ value & $<0.001$ & $<0.002$ & $<0.003$ & $<0.004$ & $<0.005$ & $<0.006$ & $<0.007$ & 0.744 & $<0.001$ & $<0.001$ & $<0.001$ \\
\hline$N$ & 83 & 83 & 83 & 83 & 82 & 83 & 83 & 83 & 83 & 83 & 83 \\
\hline \multicolumn{12}{|c|}{ Russet Norkotah } \\
\hline Pearson & $0.282 *$ & $0.484 * *$ & $0.540 * *$ & 0.136 & -0.132 & -0.178 & $0.555 * *$ & $0.422 * *$ & $0.772 * *$ & $0.812 * *$ & $0.811^{* *}$ \\
\hline$P$ value & 0.012 & $<0.001$ & $<0.002$ & 0.237 & 0.249 & 0.118 & $<0.001$ & $<0.002$ & $<0.003$ & $<0.004$ & $<0.005$ \\
\hline$N$ & 78 & 78 & 78 & 78 & 78 & 78 & 78 & 78 & 78 & 78 & 78 \\
\hline
\end{tabular}

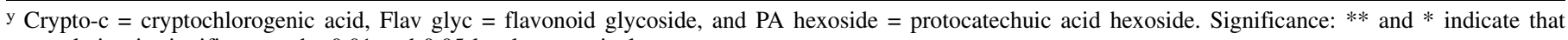
correlation is significant at the 0.01 and 0.05 level, respectively.

$\mathrm{z}$ Pearson $=$ Pearson correlation .

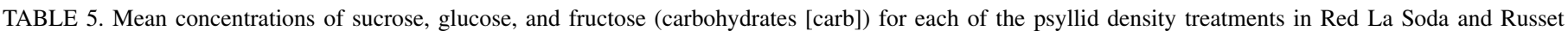
Norkotah

\begin{tabular}{|c|c|c|c|c|c|c|c|c|}
\hline \multirow[b]{3}{*}{ Cultivar, carb } & \multicolumn{8}{|c|}{ Concentration (mg/g fresh weight \pm standard error) per year and psyllid density ${ }^{z}$} \\
\hline & \multicolumn{4}{|c|}{2012} & \multicolumn{4}{|c|}{2013} \\
\hline & 0 & 5 & 15 & 30 & 0 & 5 & 15 & 30 \\
\hline \multicolumn{9}{|l|}{ Red La Soda } \\
\hline Fructose & $0.17 \pm 0.05 \mathrm{a}$ & $0.40 \pm 0.09 \mathrm{ab}$ & $0.73 \pm 0.13 b$ & $1.27 \pm 0.14 \mathrm{c}$ & $0.22 \pm 0.03 \mathrm{a}$ & $0.66 \pm 0.28 \mathrm{ab}$ & $2.38 \pm 0.87 \mathrm{~b}$ & $0.67 \pm 0.31 \mathrm{ab}$ \\
\hline Glucose & $0.68 \pm 0.13 \mathrm{a}$ & $1.47 \pm 0.28 \mathrm{ab}$ & $2.00 \pm 0.36 \mathrm{bc}$ & $2.68 \pm 0.35 \mathrm{c}$ & $0.27 \pm 0.06 \mathrm{a}$ & $0.95 \pm 0.56 \mathrm{a}$ & $1.86 \pm 0.67 \mathrm{a}$ & $0.91 \pm 0.39 b$ \\
\hline Sucrose & $2.89 \pm 0.24 \mathrm{a}$ & $2.74 \pm 0.28 \mathrm{a}$ & $3.04 \pm 0.19 \mathrm{a}$ & $2.61 \pm 0.16 \mathrm{a}$ & $6.44 \pm 0.28 \mathrm{a}$ & $8.00 \pm 0.94 \mathrm{a}$ & $6.06 \pm 0.90 \mathrm{a}$ & $3.72 \pm 0.08 b$ \\
\hline \multicolumn{9}{|c|}{ Russet Norkotah } \\
\hline Fructose & $0.11 \pm 0.02 \mathrm{a}$ & $0.89 \pm 0.12 b$ & $0.90 \pm 0.10 \mathrm{~b}$ & $0.89 \pm 0.17 b$ & $0.32 \pm 0.16 \mathrm{a}$ & $0.40 \pm 0.14 \mathrm{a}$ & $2.13 \pm 0.41 b$ & $0.32 \pm 0.13 \mathrm{a}$ \\
\hline Glucose & $0.36 \pm 0.02 \mathrm{a}$ & $0.74 \pm 0.08 b$ & $0.87 \pm 0.10 b$ & $0.92 \pm 0.13 b$ & $0.41 \pm 0.20 \mathrm{ab}$ & $0.51 \pm 0.15 \mathrm{a}$ & $0.50 \pm 0.20 \mathrm{ab}$ & $0.01 \pm 0.00 \mathrm{~b}$ \\
\hline Sucrose & $2.40 \pm 0.24 \mathrm{a}$ & $1.95 \pm 0.11 \mathrm{a}$ & $2.24 \pm 0.22 \mathrm{a}$ & $2.51 \pm 0.21 \mathrm{a}$ & $1.29 \pm 0.25 \mathrm{a}$ & $2.00 \pm 0.37 \mathrm{a}$ & $1.77 \pm 0.50 \mathrm{ab}$ & $1.37 \pm 0.45 b$ \\
\hline
\end{tabular}

$\mathrm{z}$ Different letters indicate significant differences $(P<0.05)$ across density treatments. 
differentially trigger the host's defensive physiological responses, resulting in different degrees and timing of symptom development.

One might argue that density treatments used in this study may not represent natural circumstances where infested plants may harbor only one or very few insects. However, single plant vector densities equal to or greater than those used in this study are encountered in fields where chemical control is lacking or ineffective (personal observations). The vector densities in the present experiment were selected to assure success in detecting meaningful shifts in biochemical responses, known to be highly variable and influenced by abiotic environmental factors (Brunetti et al. 2013; Rodziewicz et al. 2013). Selecting a lower density of one or two insects might not have provided a sufficient number of replicates, due to expected mortality of some of the released greenhouse-raised psyllids that were not adapted to field conditions. It was also interesting that variations in ' $C a$. L. solanacearum' titer and symptom severity were mostly detected between the 5-psyllid treatment and the 15- and 30psyllid treatments, indicating that the effect of vector number on ZC severity may level off when the total number of infective potato psyllids reaches a certain number. Our approach to remove bacteriliferous psyllids after 1 week of feeding was set to stimulate a more likely scenario of ' $\mathrm{Ca}$. L. solanacearum' infection in the field, where plants are sprayed with intensive application schedules (Goolsby et al. 2007). Although decoupling plant physiological responses to psyllid and ' $\mathrm{Ca}$. L. solanacearum' infestations was beyond the scope of this work, including nonbacteriliferous psyllid controls would provide additional insights to vector-plantpathogen interactions and should be the focus of future studies. Regardless, our approach was sensitive enough to reveal variations in ' $C a$. L. solanacearum' titer and various biochemical components across evaluated vector density treatments, and results support the need for continued late-season pest management.

The significant and positive relationship that was observed between ' $C a$. L. solanacearum' titer and symptom severity was supported by Wallis et al. (2014). However, these findings are in contrast to those of Rashed et al. (2014), which found no relationship between the two variables. It is important to note that the relationship between titer and symptom expression appears to be affected by the duration of infection. Indeed, the lack of relationship between ' $\mathrm{C} a$. L. solanacearum' titer and the severity of the symptoms in Rashed et al. (2014) was attributed to continuous and cumulative plant defense response to ' $\mathrm{Ca}$. $\mathrm{L}$. solanacearum' infection over time, which might have reduced detectable titer levels in highly symptomatic tubers of early infested plants (infested prior to and during tuber initiation stages) (Rashed et al. 2014). In relation to this, although a single psyllid is capable of infecting a plant with ' $\mathrm{Ca}$. L. solanacearum' and causing severe symptoms, given sufficient time (Buchman et al. 2011; Rashed et al. 2012), the time needed for the expression of symptoms and shifts in tuber chemistry may be highly dependent on the initial titer amount. Initial ' $C a$. L. solanacearum' titer now has been confirmed to be associated with vector density on the host plant and, therefore, constitutes an important issue when it comes to patterns and

TABLE 6. Relationships between 'Candidatus Liberibacter solanacearum' titer and sucrose, glucose, and fructose concentrations

\begin{tabular}{lccc}
\hline & \multicolumn{3}{c}{ Carbohydrates $^{\mathrm{z}}$} \\
\cline { 2 - 4 } Cultivar & Sucrose & Glucose & Fructose \\
\hline Red La Soda & & & \\
$\quad$ Pearson correlation & -0.138 & $0.425^{* *}$ & $0.459^{* *}$ \\
$P$ value & 0.202 & $<0.001$ & $<0.001$ \\
$\quad N$ & 87 & 87 & 87 \\
$\quad$ Russet Norkotah & & & \\
$\quad$ Pearson correlation & 0.031 & -0.156 & $0.388^{* *}$ \\
$P$ value & 0.777 & 0.151 & $<0.001$ \\
$N$ & 86 & 86 & 86 \\
\hline
\end{tabular}

${ }^{\mathrm{z}}$ Significance: * and $* *$ indicate that correlation is significant at the 0.05 and 0.01 level, respectively. management of late-season infestations in major potato-growing regions of the Pacific Northwest (Rush et al. 2015).

Although significant yield loss is expected in infections that occur prior to and during tuber initiation (Buchman et al. 2012; Rashed et al. 2014), the impact of ZC on harvested tuber quality is considered to be the most economically significant aspect of this disease, an effect which is known to be associated with shifts in various biochemical properties of the infected tubers (Gao et al. 2009; Navarre et al. 2009; Wallis et al.2014). Here, we showed that the number of bacteriliferous psyllids transmitting ' $C a$. L. solanacearum' can influence activity levels of some of these physiological responses associated with ZC.

In both Red La Soda and Russet Norkotah, the majority of amino acids increased in response to infection. The pattern of increase, however, did not appear to be consistently correlated with the number of infective psyllids (Table 1). Some amino acids such as asparagine are known to be the carbon source for acrylamide (Stadler et al. 2002), the product of a Maillard-driven reaction that is thought to be the cause of the burnt pattern in the fried ZC-affected tubers (Wallis et al. 2012). In this study, asparagine concentration was not affected by ' $\mathrm{Ca}$. L. solanacearum' infection in either cultivar, which was consistent with results of a previous study using a chipping cultivar (FL1867) (Rashed et al. 2013). Glutamine, another amino acid contributing to acrylamide production, was not affected by density treatment in Red La Soda but showed a significant increase in response to infection in Russet Norkotah (Table 1). It has been suggested that changes in amino acids with negatively charged chains such as aspartic acid and glutamic acid and their uncharged counterparts, asparagine and glutamine, may be associated with early stages of infection. Such amino acids that peak early may be disproportionately utilized by ' $C a$. L. solanacearum' or differentially regulated following plant response to infection (Wallis et al. 2014).

Reducing sugar concentrations showed variation in response to vector density treatments. Although the general pattern was indicative of an increasing trend in the reducing sugar concentrations in response to psyllid infestations, it was only in Red La Soda that glucose concentrations increased with an increase in psyllid numbers. However, in the case of phenolics, overall, lower activity levels were detected in plants infested with 5 infective psyllids than plants infested with 15 and 30 infective psyllids, a pattern corresponding to those of ' $\mathrm{Ca}$. L. solanacearum' titer and symptom severity. This observation not only supports results of a previous study showing significant covariation between phenolic compounds and ZC severity (Rashed et al. 2013) but also shows that shifts in plant chemistry are influenced by vector numbers. The impact of vector density, however, appears to diminish when relatively higher vector densities are present.

Although changes in amino acids and reducing sugars are also expected in response to infection, such responses may not be consistent across various cultivars with different marketing traits. To develop more tolerant cultivars, perhaps focusing on reduced phenolic production that occurs in response to ' $\mathrm{Ca}$. L. solanacearum' infection may more consistently lead to the production of less susceptible cultivars.

Currently, frequent pesticide applications have proven somewhat successful in managing ZC (Goolsby et al. 2007). Although relatively high densities of insect vectors were used in the present study, our results highlight the importance of potato psyllid control in managing ZC, because ' $\mathrm{Ca}$. L. solanacearum' titer and shifts in biochemical properties of potato tubers were affected by the number of infective psyllids, which may result in considerable quality defects, especially in plants infested later in the season. It is clear from this study that continued work on development of tolerant and resistant genotypes is essential for implementing an integrated and sustainable disease management plan for ZC.

\section{ACKNOWLEDGMENTS}

We thank our lab members for their help with different aspects of this project and the Texas Department of Agriculture Zebra Chip Initiative 
(number ZC-1213-01), USDA Specialty Crop Research Initiative (number 2009-511-20176), and USDA National Institute of Food and Agriculture Hatch-IDA01506 for funding this work. Mention of trade names or commercial products in this publication is solely for the purpose of providing specific information and does not imply recommendation or endorsement by the U.S. Department of Agriculture. USDA is an equal opportunity provider and employer.

\section{LITERATURE CITED}

Bove, J. M. 2006. Huanglongbing: A destructive, newly emerging, century old disease of citrus. J. Plant Pathol. 88:7-37.

Brunetti, C., George, R. M., Tattini, M., Field, K., and Davey, M. P. 2013. Metabolomics in plant environmental physiology. J. Exp. Bot. 64:4011-4020.

Buchman, J. L., Fisher, T. W., Sengoda, V. G., and Munyaneza, J. E. 2012. Zebra chip progression: From inoculation of potato plants with Liberibacter to development of disease symptoms in tubers. Am. J. Potato Res. 89:159-168.

Buchman, J. L., Sengoda, V. G., and Munyaneza, J. E. 2011. Vector transmission efficiency of Liberibacter by Bactericera cockerelli (Hemiptera: Triozidae) in zebra chip potato disease: Effects of psyllid life stage and inoculation access period. J. Econ. Entomol. 104:1486-1495.

Gao, F., Jifon, J., Yang, X. B., and Liu, T. X. 2009. Zebra chip disease incidence on potato is influenced by timing of potato psyllid infestation, but not by the host plants on which they were reared. Insect Sci. 16:399-408.

Goolsby, J. A., Adamczyk, J., Bextine, B., Lin, D., Munyaneza, J. E., and Bester, G. 2007. Development of an IPM program for management of the potato psyllid to reduce incidence of zebra chip disorder in potatoes. Subtrop. Plant Sci. 59:85-94.

Hansen, A. K., Trumble, J. T., Stouthamer, R., and Paine, T. D. 2008. A new huanglongbing species, 'Candidatus Liberibacter psyllaurous,' found to infect tomato and potato, is vectored by psyllid Bactericera cockerelli (Sulc). Appl. Environ. Microbiol. 74:5862-5865.

Li, W., Abad, J. A., French-Monar, R. D., Rascoe, J., Wen, A., Gudmestad, N. C., Secor, G. A., Lee, I., Duan, Y., and Levy, L. 2009. Multiplex real-time PCR for detection, identification and quantification of 'Candidatus Liberibacter solanacearum' in potato plants with zebra chip. J. Microbiol. Methods 78:59-65.

Li, W., Hartung, J. S., and Levy, L. 2006. Quantitative real-time PCR for detection and identification of Candidatus Liberibacter species associated with citrus huanglongbing. J. Microbiol. Methods 66:104-115.

Lopes, S. A., Frare, G. F., Bertolini, E., Cambra, M., Fernandes, N. G., Ayres, A. J., Martin, D. R., and Bove, J. M. 2009. Liberibacter associated with citrus huanglongbing in Brazil: 'Candidatus Liberibacter asiaticus' is heat tolerant, ' $C a$. L. americanus' is heat sensitive. Plant Dis. 93:257-262.

Munyaneza, J. E. 2012. Zebra chip disease of potato: Biology, epidemiology and management. Am. J. Potato Res. 89:329-350.

Munyaneza, J. E., Crosslin, J. M., and Upton, J. E. 2007. Association of Bactericera cockerelli (Homoptera: Psyllidae) with "Zebra Chip," a new potato disease in southwestern United States and Mexico. J. Econ. Entomol. 100:656-663.

Munyaneza, J. E., Sangoda, V. G., Buchman, J. L., and Fisher, T. W. 2012. Effects of temperature on 'Candidatus Liberibacter solanacearum' and zebra chip potato disease symptom development. Plant Dis. 96:18-23.

Navarre, D. A., Shakya, R., Holden, J., and Crosslin, J. M. 2009. LC-MS analysis of phenolic compounds in tubers showing zebra chip symptoms. Am. J. Potato Res. 86:88-95.
Rashed, A., Nash, D., Paetzold, L., Workneh, F., and Rush, C. M. 2012. Transmission efficiency of 'Candidatus Liberibacter solanacearum' and potato zebra chip disease progress in relation to pathogen titer, vector numbers and feeding sites. Phytopathology 102:1079-1085.

Rashed, A., Wallis, C. M., Paetzold, L., Workneh, F., and Rush, C. M. 2013. Zebra chip disease and potato biochemistry: Tuber physiological changes in response to 'Candidatus Liberibacter solanacearum' infection over time. Phytopathology 103:419-426.

Rashed, A., Workneh, F., Gray, J., Paetzold, L., and Rush, C. M. 2014. Zebra chip disease development in relation to plant age and time of 'Candidatus Liberibacter solanacearum' infection. Plant Dis. 98:24-31.

Rodziewicz, P., Swarcewicz, B., Chmielewska, K., Wojakowska, A., and Stobiecki, M. 2013. Influence of abiotic stresses on plant proteome and metabolome changes. Acta Physiol. Plant. 36:1-19.

Rush, C. M., Workneh, F., and Rashed, A. 2015. Significance and epidemiological aspects of late-season infections in the management of potato zebra chip. Phytopathology 105:929-936.

Secor, G. A., Rivera, V. V., Abad, J. A., Lee, I. M., Clover, G. R. G., Liefting, L. W., Li, X., and De Boer, S. H. 2009. Association of 'Candidatus Liberibacter solanacearum' with zebra chip disease of potato established by graft and psyllid transmission, electron microscopy, and PCR. Plant Dis. 93: 574-583.

Stadler, R. H., Blank, I., Varga, N., Robert, F., Hau, J., Guy, P. A., Robert, M. C., and Riediker, S. 2002. Acrylamide from Maillard reaction products. Nature 419:449-450.

Swisher, K. D., Munyaneza, J. E., and Crosslin, J. M. 2012. High resolution melting analysis of the cytochrome oxidase I gene identifies three haplotypes of the potato psyllid in the United States. Environ. Entomol. 41: 1019-1028.

Wallis, C. M., Chen, J., and Civerolo, E. L. 2012. Zebra chip-diseased potato tubers are characterized by increased levels of host phenolics, amino acids, and defense-related proteins. Physiol. Mol. Plant Pathol. 78:66-72.

Wallis, C. M., Munyaneza, J. E., Novy, R., Bester, G., Buchman, J. L., Nordgaard, J., and van Hest, P. 2015a. 'Candidatus Liberibacter solanacearum' titers in and infection effects on potato tuber chemistry of promising germplasm exhibiting tolerance to zebra chip disease. Phytopathology 105:1573-1584.

Wallis, C. M., Rashed, A., Chen, J., Paetzold, L., Workneh, F., and Rush, C. M. 2015b. Effects of potato-psyllid-vectored 'Candidatus Liberibacter solanacearum' infection on potato leaf and stem physiology. Phytopathology 105:189-198.

Wallis, C. M., Rashed, A., Paetzold, L., Workneh, F., and Rush, C. M. 2014. Similarities and differences in physiological responses to 'Candidatus Liberibacter solanacearum' infection among different potato cultivars. Phytopathology 104:126-133.

Wen, A., Lin, H., and Gudmestad, N. C. 2012. Development of PCR assay using SSR primers for detection and genotyping of 'Candidatus Liberibacter solanacearum'. Pages 74-78 in: Proc. 11th Annu. SCRI Zebra Chip Rep. Sess. F. Workneh, A. Rashed, and C. M. Rush, eds. Fredric Printing, Aurora, CO.

Wen, A., Mallik, I., Alvarado, V. Y., Pasche, J. S., Wang, X., Li, W., Levy, L., Lin, H., Scholthof, H. B., Mirkov, T. E., Rush, C. M., and Gudmestad, N. C. 2009. Detection, distribution, and genetic variability of 'Candidatus Liberibacter' species associated with zebra chip complex disease 1 of potato in North America. Plant Dis. 93:1102-1115. 